\title{
ROLE OF MRI IN EVALUATION OF TRAUMATIC KNEE INJURIES
}

Saurabh Chaudhuri, Priscilla Joshi, Mohit Goel

1. Associate Professor, Department of Radiodiagnosis \& imaging, Bharati Vidyapeeth Medical College and Hospital, Pune.

2. Professor, Department of Radiodiagnosis \& imaging, Bharati Vidyapeeth Medical College and Hospital, Pune.

3. Resident, Department of Radiodiagnosis \& imaging, Bharati Vidyapeeth Medical College and Hospital, Pune.

\section{CORRESPONDING AUTHOR}

Dr. Saurabh Chaudhuri,

Row House No. 37,

Hill View Society,

Ramnagari , Ambegaon,

Pune 411046.

E-mail: saurabhplus@gmail.com

Ph: 00919373322501

ABSTRACT: MRI is an accurate, noninvasive imaging modality for evaluation of knee injuries, and determines the patient management, saving them from unnecessary arthroscopy. Our study focuses on the fundamental biomechanical forces underlying the most common injuries and correlate MRI findings with specific traumatic mechanisms. We conducted a retrospective study on 138 patients having prior history of knee trauma. The studies evaluated cruciate ligament tears, collateral ligament injuries, chondromalacia patella, bone marrow contusions, joint effusions, tendon tear, meniscal tear and osteoarthritis.

KEYWORDS: MRI, biomechanical forces, knee, ligament injuries, chondromalacia patella, bone marrow contusion, joint effusion.

INTRODUCTION: Since the initial application in 1984, Magnetic resonance (MR) imaging of the knee has undergone significant advances for evaluation of the menisci, ligaments, bone marrow contusions. MR examination, a noninvasive modality, is now routinely used to assess a wide spectrum of internal knee derangements and articular disorders and has virtually replaced conventional arthrography in the evaluation of the menisci and the cruciate ligaments. Biomechanical forces play a very vital role in ascertaining the type of knee injuries. In addition to diagnostic benefits and in the selection of surgical candidates with preoperative planning MR imaging has proved valuable. Further, improved patient-doctor communication and reduction in the cost of MR knee studies has contributed to their greater acceptance by the orthopaedic community. MRI has become the imaging modality of choice for the evaluation of the painful knee following injury. It can detect soft tissue abnormalities (meniscal, cruciate, collateral ligament tears) and microtrabecular fractures that cannot be detected by plain film.

METHODS: The study consisted of 138 adult cases which were referred to the Department of Radiodiagnosis and Imaging, Bharati Hospital, over a period of 7 months from May-2011 to April 2012. All patients with prior history of knee trauma were subjected to MRI on a 1.5 Tesla Philips ACHIEVA MRI machine. A detailed history of biomechanics of trauma was taken. Fast spin-echo (FSE) imaging in conjunction with fat suppression (FS) MR techniques, were used 
which extended the sensitivity and specificity of MR in the detection of articular cartilage and ligament injuries.

STATISTICS: Out of the studied 138 adult cases [Table 1, Figure 1,2] ; Joint effusion is the most common finding, followed by ACL tear and medial meniscal injury. MCL injury was found to be higher than LCL injury. PCL tear was relatively less common. The biomechanics of injury is discussed in detail in [Table 2].

RESULTS: MRI has become the preferred imaging technique for the evaluation of the painful knee following injury. It can detect soft tissue abnormalities (meniscal and cruciate/collateral ligament tears) and fractures that cannot be detected by plain film. ACL tears were the most common injuries as observed in our study. Another interesting finding was that ACL tear were invariably accompanied by joint effusions. Most common biomechanics of trauma involved in ACL injury is external rotation, PCL injury is hyperextension, collateral ligament is direct trauma, medial meniscal injury is twisting, and lateral meniscus injury is internal rotation.

DISCUSSION: Magnetic Resonance Imaging (MRI) has gained popularity as a diagnostic tool for evaluation of musculoskeletal disorders. The knee is the most frequently examined joint. Traumatic injury to the knee remains a diagnostic and therapeutic challenge.

Tear of the anterior cruciate ligament (ACL) [Figure 3] - is one of the most common injuries to the knee [1]. The biomechanics involved in ACL tear are - External rotation and abduction with hyperextension; direct forward displacement of the tibia; internal rotation with the knee in full extension. ACL tear is commonly seen in- Pivot shift injury (Noncontact injury in skiers or football player), Dashboard injury (force to anterior proximal tibia with knee in flexion), Hyperextension injury (force applied to the anterior tibia with a planted foot), Clip injury (Contact injury secondary to pure valgus stress to a partially flexed knee). A torn ACL may be demonstrated on MR images by the absence or abnormal course of the ligament or by high signal intensity fluid traversing the ligament on a T2-weighted sequence. The most common site of a tear is near the femoral attachment [2].

The PCL is twice as strong as the ACL. PCL tears lag behind that of anterior cruciate ligament (ACL) tears. The biomechanics involved in PCL tear are - Excessive rotation; hyperextension; dislocation; direct trauma while the knee is flexed [3]. PCL tear is commonly seen in- motor vehicle accidents (dashboard injuries) and injuries sustained in contact sports such as football injuries to the PCL are usually associated with tears of the ACL, the meniscus, collateral ligaments, or posterolateral structures [4],[5]. PCL tears are identified on PD fat sat sagittal T1-weighted images by discontinuity of the ligament and are more obvious on T2weighted images due to the high signal intensity of fluid within the tear [6]. Avulsion of the ligament from its tibial attachment is identified on MR images by bony fracture of the posterior tibial plateau and redundancy of the ligament.

Meniscal injury may be associated with a history of twisting, squatting, or cutting [7].The biomechanics involved in meniscal injuries are the rotation of the femur against a fixed tibia during flexion and extension places the menisci at risk for injury. The normal meniscus is homogeneously black (devoid of signal). A meniscal tear [Figure 4] - is identified on MR images by the presence of an intrameniscal signal that may extend to the meniscal surface.

The medial collateral ligament complex is most commonly injured by a valgus stress due to a direct blow to the lateral aspect of the knee [8]. Normal medial and lateral 
ligament complexes are visualized as low signal intensity bands on both T1- and T2-weighted images. Injuries are identified by increased signal intensity due to edema and hemorrhage, increased thickness, abnormal configuration, and/or discontinuity of the ligament.

Bone marrow edema/contusions/bruising [Figure 5] - are also called "trabecular fractures". The pattern of bone marrow edema is like a footprint left behind at the site of injury [9]. Bone bruising has been increasingly identified in association with soft tissue knee injury, in particular anterior cruciate ligament rupture. These cannot be seen on plain films and can be a major source of pain [10]. Because their water content is higher than that of adjacent fatty bone marrow, these contusions are best detected on fat-suppressed T2-weighted images [11]. Continued activity in this setting could convert a bone bruise with intact cartilage to one in which the cartilage becomes disrupted, leading to early osteoarthritis [12].

With advancing osteoarthritis, the articular cartilage is completely denuded and subchondral sclerosis can be seen as low intensity on all sequences. With continued bone-onbone irritation, fibro-vascular reaction can lead to bone marrow edema, which appears bright on STIR or fat-saturated T2-weighted fast spin-echo images.

Joint effusion [Figure 3,6] - Bone marrow contusions or fractures associated with cortical disruption can leak marrow into the joint space, leading to a lipohemarthrosis. Since sagittal images are acquired with the patient supine, fat rises to the top, joint fluid is in the middle, and intact red cells are at the bottom.

Most patients with chondromalacia patellae have focal increased signal in the cartilage or focal contour defects in the cartilage surface on T2-weighted MR images [13].

CLINICAL RELEVANCE / APPLICATION: MRI is an extremely useful imaging modality for evaluation of knee injuries. It gives valuable information to the referring orthopaedician for planning the line of treatment in terms of conservative management or surgery. MRI is not only a diagnostic tool but also helps in correlation of injury with biomechanical forces involved in trauma and follow up post-operative patients.

\section{REFERENCES}

1. Turner DA, Prodromos CC, Pet snick JP, Clark JW (1985). Acute injury of the ligaments of the knee: magnetic resonance evaluation. Radiology 166: 865.

2. Turner DA, Prodromos CC, Pet snick JP, Clark JW (1985). Acute injury of the ligaments of the knee: magnetic resonance evaluation. Radiology 166: 865.

3. Miller MD, Harner CD. Posterior cruciate ligament injuries. Current concepts in diagnosis and treatment. Physician Sports Med 1993;21:38.

4. Clancy WG. Treatment of knee joint instability secondary to rupture of the posterior cruciate ligament: report of a new procedure. J Bone Joint Surg [Am] 1983;65:310.

5. Hughston JC. Acute tears of the posterior cruciate ligament: results of operative treatment. J Bone Joint Surg [Am] 1980;62:438.

6. Grover JS, Bassett LW, Gross ML, Seeger LL, Finermann GAM (1990). MR imaging of the posterior cruciate ligament. Radiology 174:527.

7. Cannon WD, Morgan CD. Meniscal repair: II. Arthroscopic repair techniques. J Bone Joint Surg [Am] 1994;76:294.

8. Grover JS, Bassett LW, Gross ML, Seeger LL, Finermann GAM (1990). MR imaging of the posterior cruciate ligament. Radiology 174:527. 
9. Graf BK, Cook DA, De Smet AA, Keene JS. "Bone bruises" on magnetic resonance imaging evaluation of anterior cruciate ligament injuries. Am J Sports Med 1993; 21:220-223.

10. Andrish JT (1985) Ligamentous injuries of the knee. Orthop Clin North Am 16:273.

11. Kapelov SR, Teresi LM, Bradley WG, et al. Bone contusions of the knee: Increased lesion detection with fast spin-echo MR imaging with spectroscopic fat saturation. Radiology.1993;189:901-904.

12. Andrish JT (1985) Ligamentous injuries of the knee. Orthop Clin North Am 16:273.

13. Thomas R. McCauley1, Ruben Kier1, Kevin J. Lynch2, Peter Jokl2. Chondromalacia Patellae: Diagnosis with MR Imaging. AJR 158:101-105, January 1992.

TABLE 1: Distribution of total number of cases.

\begin{tabular}{|c|l|l|l|}
\hline Sr. No & OBSERVATIONS & $\underline{\text { CASES [OUT OF 138 ] }}$ & PERCENTAGE \% \\
\cline { 2 - 3 } 1 & ACL tear & 71 & 51 \\
2 & PCL tear & 14 & 10 \\
3 & MCL injury & 21 & 15 \\
4 & LCL injury & 17 & 12 \\
5 & Chondromalacia patella & 19 & 14 \\
6 & Bone marrow edema/ contusion & 53 & 38 \\
7 & Joint effusion & 104 & 75 \\
8 & Tendon tear & 6 & 4 \\
9 & Medial meniscus tear & 66 & 48 \\
10 & Lateral meniscus tear & 28 & 20 \\
11 & Osteoarthritis & & 17 \\
\hline
\end{tabular}

TABLE 2: Correlation of biomechanics involved with different types of injuries

\begin{tabular}{|c|c|c|c|c|c|c|}
\hline BIOMECHANICS & ACL INJURY & PCL INJURY & MCL INJURY & LCL INJURY & $\begin{array}{c}\text { MEDIAL } \\
\text { MENISCUS } \\
\text { INJURY }\end{array}$ & $\begin{array}{c}\text { LATERAL } \\
\text { MENISCUS } \\
\text { INJURY }\end{array}$ \\
\hline External Rotation & 39 & 2 & 6 & 0 & 25 & - \\
\hline Hyperextension & 33 & 8 & 4 & 5 & 3 & - \\
\hline Internal Rotation & 25 & - & - & 3 & - & 16 \\
\hline Direct Trauma & 7 & 3 & 11 & 9 & 2 & - \\
\hline Twisting & 11 & 1 & - & - & 33 & 10 \\
\hline Squatting & - & - & - & - & 7 & 2 \\
\hline
\end{tabular}


ORIGINAL ARTICLE

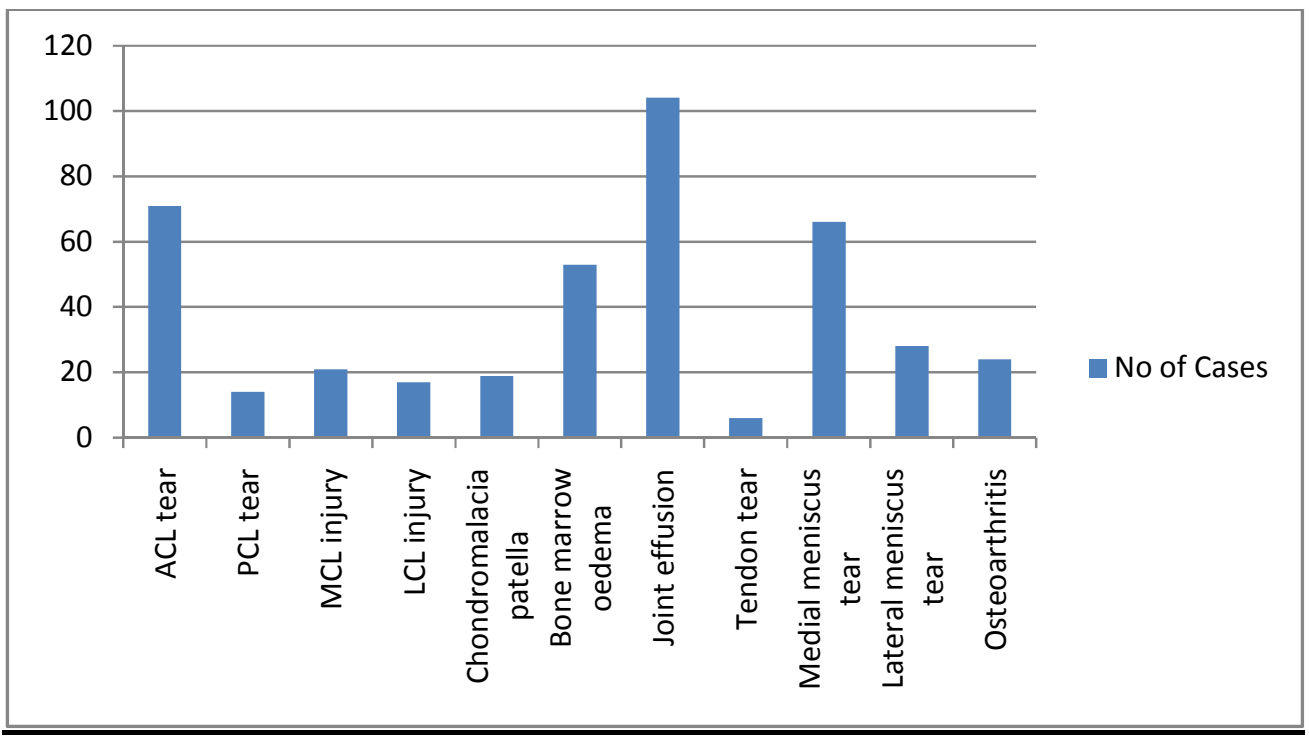

FIGURE 1: Histogram showing number of cases of different pathologies.

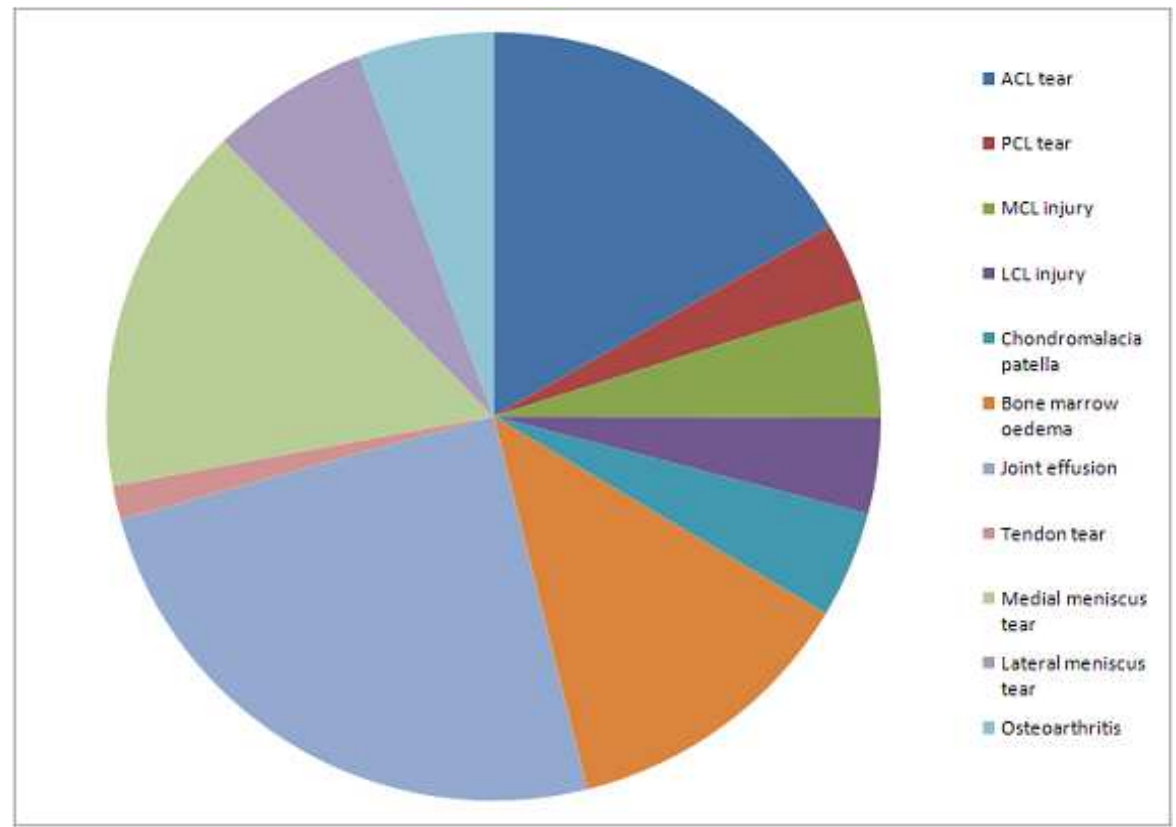

FIGURE 2: Pie chart showing number of cases of different pathologies. 


\section{ORIGINAL ARTICLE}

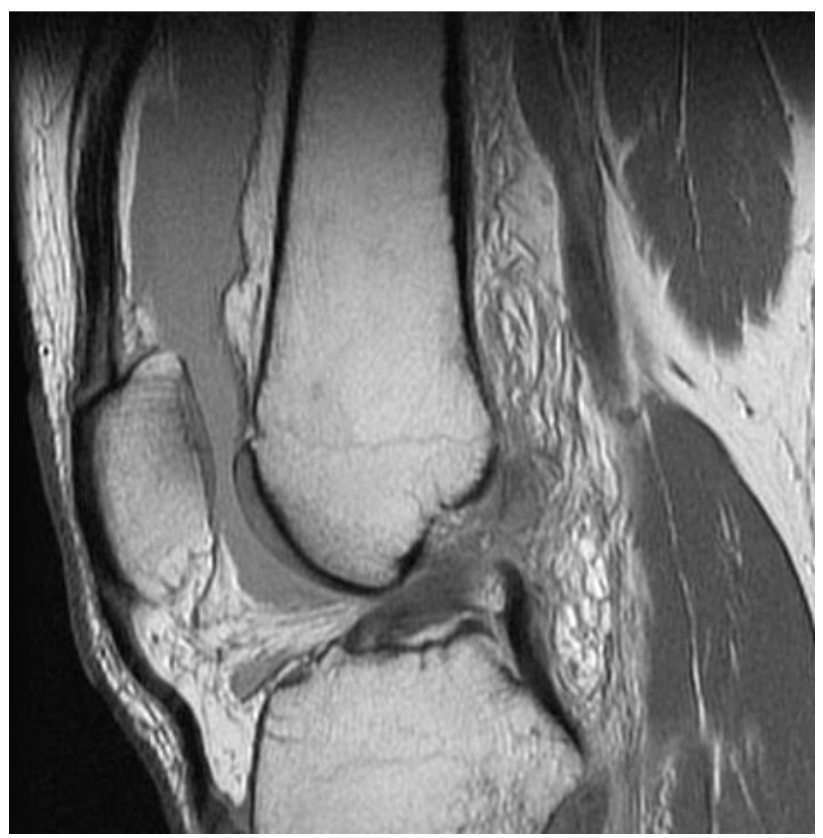

FIGURE 3: Sagittal view of the knee demonstrates an ACL tear with associated joint effusion.

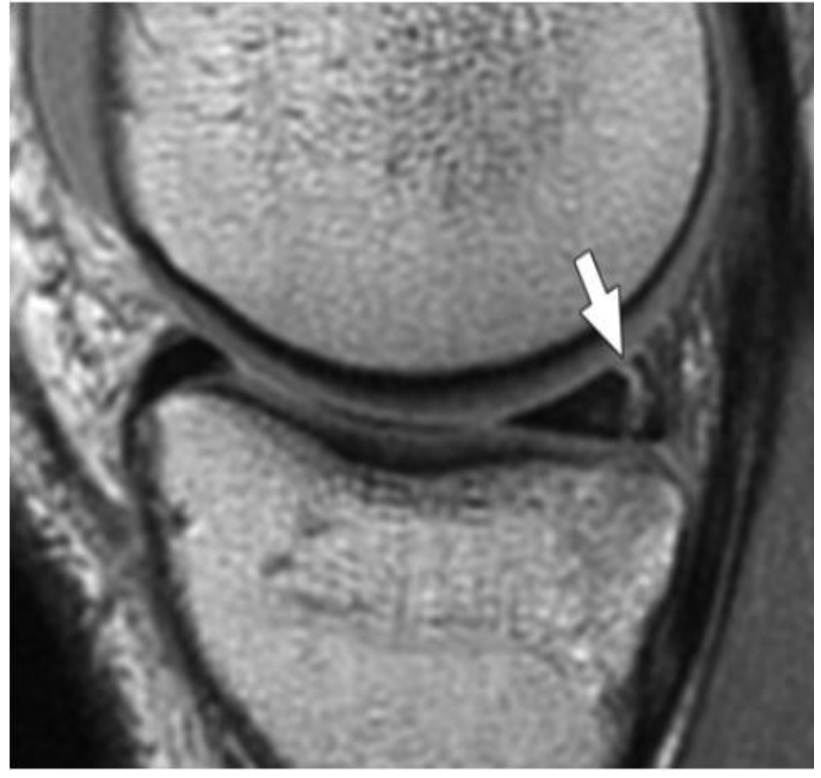

FIGURE 4: Sagittal proton density-weighted image show linear intermediate-signalintensity line (arrow) contacting superior surface of meniscus with probable inferior contact as well. 


\section{ORIGINAL ARTICLE}

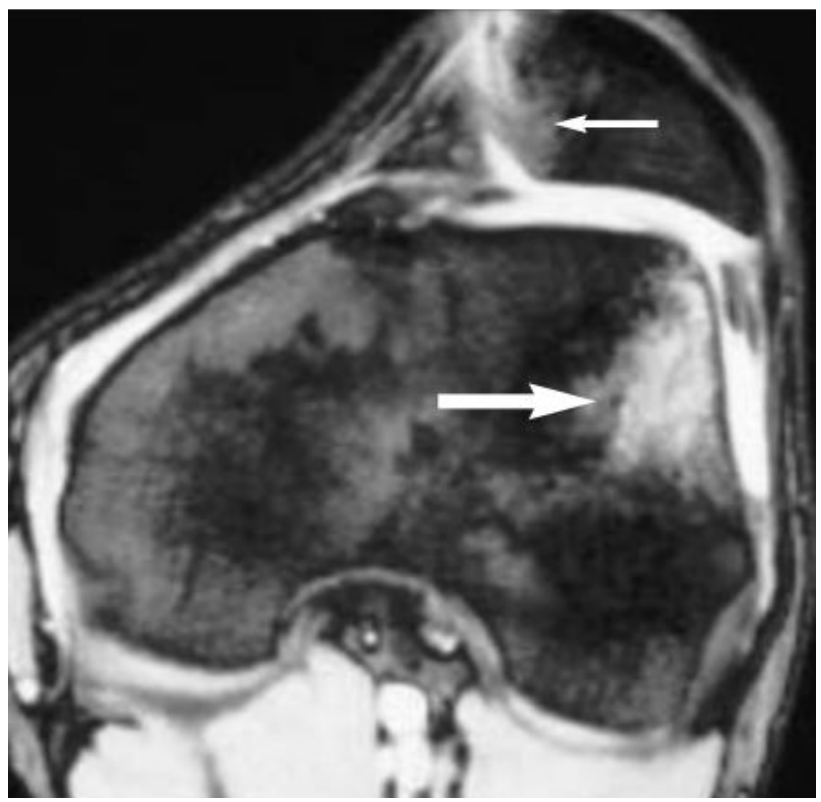

FIGURE. 5: T2-weighted axial fat-saturated fast spin-echo image reveals bone marrow edema in the lateral femoral condyle (large arrow) and in the medial patellar facet (small arrow), ie. the points of contact at the time of dislocation.

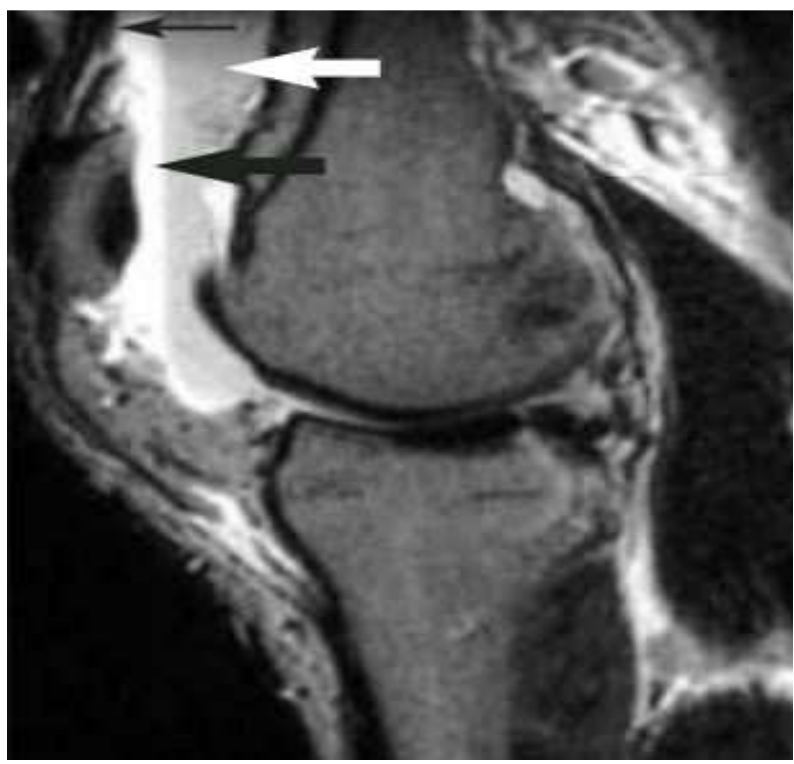

FIGURE. 6: Lipohemarthrosis. A three-layer lipohemarthrosis is seen on T2-weighted image. Specifically, note the relatively dark area in the dependent (posterior) position representing intact red cells containing paramagnetic deoxyhemoglobin (white arrow). Above this is the proteinaceous joint effusion (large black arrow); above that is a very small dark area of fat (small arrow). 